\title{
Approximations of continuous functions by squares
}

\author{
PAUL D. HUMKE†
}

Department of Mathematics, St Olaf College, Northfield, MN 55057, USA

\author{
MIKLós LACZKOVICH
}

Eötvös Loränd University, Department of Analysis, Budapest, Múzeum krt. 6-8, Hungary, $\mathrm{H}-1088$

(Received 19 April 1988)

\begin{abstract}
Let $C$ denote the space of continuous functions mapping $[0,1]$ into itself and endowed with the sup metric. It has been shown that $C^{2}=\{f \circ f: f \in C\}$ is an analytic but non-Borel subset of $C$. This implies that there is no simple geometric characterization for a function being a square. In this paper we consider the problem of characterizing those functions which can be approximated by squares. In the first section we prove that any continuous function mapping a closed proper subset of $[0,1]$ into $[0,1]$ can be extended to a square. In particular this shows that $C^{2}$ is $L_{p}$ dense in $C$. On the other hand, $C^{2}$ does not contain a ball when $C$ is endowed with the sup metric. In the second section we prove that no strictly decreasing function can be uniformly approximated by squares, although the distance between the class of strictly decreasing functions and $C^{2}$ is zero. In the last section we investigate the function $f(x)=1-x$ and show that $\left\|g^{2}-f\right\|>\frac{1}{4}$ for every $g \in C$ and that $\frac{1}{4}$ cannot be improved.
\end{abstract}

\section{Elementary observations}

First we prove the aforementioned result [1] concerning extending a given function to a square.

THEOREM 1. Let $F$ be a closed proper subset of $[0,1]$ and let $f: F \rightarrow[0,1]$ be continuous. Then there is a $g \in C$ such that $f=g^{2} \mid F$.

Proof. Let $[c, d] \subset[0,1] \backslash F$ and let $g$ be a homeomorphism of $F$ into [c,d]. Define $g(x)=f\left(g^{-1}(x)\right)$ whenever $x \in g(F)$. Then this extended function is continuous on $F \cup g(F)$ and hence, can be extended to a continuous function, also denoted by $g$, in $C$. It is easy to check that $g^{2} \mid F=f$.

Corollary 2. For each $p, C^{2}$ is $L^{p}$ dense in $C$.

Proof. If $f \in C$, then $f\left|[\varepsilon, 1]=g_{\varepsilon}^{2}\right|[\varepsilon, 1]$ for some $g_{\varepsilon} \in C$ by Theorem 1. Clearly, $\lim _{\varepsilon \rightarrow 0} g_{\varepsilon}^{2}=f$ in $L^{p}$. 
In the remainder of the paper we will consider $C$ to have the sup metric exclusively. We denote the closure of a set $A \subset C$ by $\bar{A}$.

THEOREM 3. If $f \in C$ with $f(0)=0$ or $f(1)=1$, then $f \in \overline{C^{2}}$.

Proof. Suppose $f \in C$ with $f(0)=0$, and let $\varepsilon>0$ be given. Then there is a $0<\delta<\varepsilon$ such that $|f(x)|<\varepsilon$ whenever $|x|<\delta$. First we choose an $f_{1} \in C$ such that $f_{1}(x)=x$ in $[0, \delta], f_{1}(x) \geq \delta$ in $[\delta, 1]$, and $\left\|f-f_{1}\right\|<\varepsilon$. Now define

$$
g(x)= \begin{cases}f_{1}\left(1-\frac{1-\delta}{\delta} x\right) & \text { for } x \in[0, \delta] \\ \frac{\delta}{1-\delta}(1-x) & \text { for } x \in[\delta, 1]\end{cases}
$$

As $f_{1}(\delta)=\delta$ it is easy to check that $g \in C$. If $x \in[0, \delta]$, then $g(x) \geq \delta$ because $1-[(1-\delta) / \delta] x \geq \delta$ and $f_{1}(x) \geq \delta$ for $x \in[\delta, 1]$. Hence, $g^{2}(x) \leq \delta$ whenever $x \in[0, \delta]$ so that $\left|g^{2}(x)-f(x)\right|<\varepsilon$ in this interval. If $x \in[\delta, 1]$, then $g^{2}(x)=f_{1}(x)$ and hence $\left|g^{2}(x)-f(x)\right|<\varepsilon$ everywhere. The case in which $f(1)=1$ is handled similarly; this completes the proof.

We denote the range of a function $f$ by $R(f)$.

THEOREM 4. Let $f \in C$ and suppose $f \mid[a, b]=c$ where $(a, b) \cap R(f) \neq \varnothing$ and $f(c) \neq c$. If $f=g^{2}$ then there is an interval $I$ and $a d \neq c$ such that $f \mid I=d$.

Proof. If $g$ is constant on $[a, b]$ we let $d$ be this constant and set $J=g^{-1}((a, b))$. Since $R(f) \subset R(g), J$ is open and nonempty. Obviously,

$$
f(J)=g(g(J)) \subset g((a, b))=\{d\} .
$$

As $f^{2}=g$, we must have $g(d)=c$. If $c=d$, then $c=g(c)=g^{2}(c)$ which is impossible. Hence $d \neq c$ and this proves our assertion in this case. Suppose instead that $g([a, b]) \equiv I$ is an interval. We put $d=g(c)$. Then

$$
f(I)=g^{2}(I)=g^{3}([a, b])=g(c)=d .
$$

As $c$ is not a fixed point of $f$ it follows that $d \neq c$ and the theorem is proved.

Corollary 5. $C^{2}$ does not contain a ball.

Proof. We use Theorem 4 to show that every given ball contains a function which is not a square. Let $B \subset C$ be a ball and let $g \in B$ be nonconstant. Suppose $(a, b) \subset$ $R(g)$. It is easy to see that $B$ contains a function $f$ such that $f$ is constant on a subinterval of $(a, b)$ which meets $R(f)$, this constant is not a fixed point of $f$ and $f$ is not constant on any other interval. It is a consequence of Theorem 4 that such a function is not a square.

\section{2}

In this section we prove that $C^{2}$ is not everywhere dense in $C$. If $f \in C$, we denote the $\max \{f(x): 0 \leq x \leq 1\}$ by $M(f)$ and the $\min \{f(x): 0 \leq x \leq 1\}$ by $m(f)$. It is easy to see that both $M(f)$ and $m(f)$ are continuous functions of $f$ as maps from $C$ into $[0,1]$. We show that the strictly decreasing functions are in the interior of 
$C \backslash C^{2}$. To establish this result, we first introduce a subclass, $\mu$, of $C$ as follows. We put $f \in M$ if $f \in C$ and there exist $0 \leq p<q<r \leq s<t<u \leq 1$ such that

1. $f(p)=M(f)$ and $f(y)<M(f)$ for $y>p$. $f(u)=m(f)$ and $f(y)>m(f)$ for $y<u$.

2. $m(f)<q$ and $M(f)>t$.

3. $r=\min \{x: f(x)=x\}$ and $s=\max \{x: f(x)=x\}$.

4. $f(x)>s$ for $x \in[0, q]$, and $f(x)<r$ for $x \in[t, 1]$.

5. $f([m(f), M(f)]) \nsubseteq f([q, t])$.

LEMMA 6. $\mathcal{M}$ is open and $\mathcal{M} \cap C^{2}=\varnothing$.

Proof. Let $f \in \mathcal{M}$ be fixed and suppose $p, q, r, s, t, u, m(f)$ and $M(f)$ are as above. Since $M(g)$ and $m(g)$ are continuous functions of $g \in C$, if $\|g-f\|$ is sufficiently small then $m(g)<q, M(g)>t$, and $g([m(g), M(g)]) \nsubseteq g([q, t])$. Also, it follows from condition 1 (above) that if $\|g-f\|$ is small enough then

$$
\begin{aligned}
& p^{\prime}=\max \{x: g(x)=M(g)\}<q, \quad \text { and } \\
& u^{\prime}=\min \{x: g(x)=m(g)\}>t .
\end{aligned}
$$

As $r$ is the first fixed point of $f$ it follows that $f(x)>x$ for $x<r$. This implies that if $\|g-f\|$ is sufficiently small, then

$$
\begin{aligned}
& r^{\prime}=\min \{x: g(x)=x\}>q, \quad \text { and } \\
& s^{\prime}=\max \{x: g(x)=x\}<t .
\end{aligned}
$$

That is, if $\|g-f\|$ is small enough, then $g$ satisfies conditions 1 through 5 for the sextuple $p^{\prime}, q, r^{\prime}, s^{\prime}, t, u^{\prime}$, and hence $\mathcal{M}$ is open.

Now suppose $\mathcal{M} \cap C^{2} \neq \varnothing$ and let $f=g^{2} \in \mathcal{M}$ where $g \in C$. As $f \in \mathcal{M}$, it satisfies conditions 1 through 5 for, say, $p, q, r, s, t$, and $u$. As every fixed point of $g$ is also a fixed point of $f$ all the fixed points of $g$ are in the interval, $[r, s]$ so that $g(x)>x$ for $x<r$ and $g(x)<x$ for $x>s$. If we let $g(u)=w$, then $w<u$.

Suppose $w>t$. Then $g(w)=f(u)=m(f) \leq r$ and $g(u)=w>t>s$. Therefore $[r, s] \subset[g(w), g(u)] \subset g([w, u])$. Let $x_{0}$ be a fixed point of $g$. Then $x_{0} \in[r, s]$ and, as a consequence, there is a $y \in[w, u]$ with $g(y)=x_{0}$. However,

$$
f(y)=g\left(x_{0}\right)=x_{0} \geq r,
$$

and this contradicts condition 4 .

Next suppose $w<q$. Then $g(w)=f(u)=m(f)<q<r$ by condition 2 , and $g(g(w))=f(w)>s$ by condition 4 . Hence,

$$
[r, s] \subset[g(w), f(w)] \subset g([w, g(w)]) .
$$

As before, there is a fixed point, $x_{0}$, of $g$ in $[r, s]$ and hence, there is a $y \in[w, g(w)]$ with $g(y)=x_{0}$. But then,

$$
f(y)=g^{2}(y)=g\left(x_{0}\right)=x_{0} \leq s
$$

which again contradicts condition 4 . 
We have proved that $w=g(u) \in[q, t]$ and by symmetry we also have that $g(p) \in$ $[q, t]$. Thus, setting $m(f)=m$ and $M(f)=M$, we obtain

$$
\begin{aligned}
M & =f(p)=g^{2}(p) \in g([q, t]), \quad \text { and } \\
m & =f(u)=g^{2}(u) \in g([q, t]) .
\end{aligned}
$$

Hence $[m, M] \subset g([q, t])$ and $g([m, M]) \subset f([q, t])$. However,

$$
f([m, M])=g(g([m, M])) \subset g(f([q, t])) \subset g([m, M]) \subset f([q, t]) .
$$

This contradiction to condition 5 completes the proof.

THEOREM 7. If $f \in C$ is strictly decreasing, then $f \notin \overline{C^{2}}$.

Proof. Let $f \in C$ be strictly decreasing. By Lemma 6 it is enough to show that $f \in \mathcal{M}$. We take $p=0, u=1$, and let $r=s$ be the only fixed point of $f$. It is easy to see that if $q \in(f(1), r)$ and $t \in(s, f(0))$, then $f$ satisfies conditions 1 through 5 .

THEOREM 8. Let $\mathscr{B}=\{f \in C: f$ is strictly decreasing, $f(0)=1$, and $f(1)=0\}$. Then $\overline{\mathscr{B}} \cap \overline{C^{2}}=\varnothing$ and $\operatorname{dist}\left(\mathscr{B}, C^{2}\right)=0$.

Proof. First we show that $\overline{\mathscr{B}} \subset \mathcal{M}$ and hence $\overline{\mathscr{B}} \cap \overline{C^{2}}=\varnothing$. Indeed, let $f \in \overline{\mathscr{B}}$, then $f$ is decreasing. Let $p=\max \{x: f(x)=1\}, u=\min \{x: f(x)=0\}$, and let $r=s$ be the unique fixed point of $f$. It is easy to see that if $p<q<\min \{x: f(x)=r\}$ and $\max \{x: f(x)=s\}<t<u$ then $f$ satisfies conditions 1 through 5 .

Next we show dist $\left(\mathscr{B}, C^{2}\right)=0$. Let $0<\varepsilon<\frac{1}{4}$ be fixed and define $f$ as follows. Set $f(0)=1-2 \varepsilon, f(1)=2 \varepsilon, f(\varepsilon)=\frac{1}{2}=f(1-\varepsilon)$, and define $f$ linearly in the intervening intervals. Then $f$ is locally constant at each point of its range and hence by Theorem 17 of [1], $f \in C^{2}$. Obviously, dist $(\mathscr{B}, f)=2 \varepsilon$ and this observation completes the proof.

3

In this section we investigate the best possible square approximation to the function $\phi(x)=1-x$. In specific we find that the distance between $\phi(x)$ and $C^{2}$ is $\frac{1}{4}$ and that this distance is not realized by any particular square. We begin with an example.

THEOREM 9. For every $0<\varepsilon<\frac{1}{4}$ there exists an $f \in C^{2}$ such that $\|f-\phi\|=\frac{1}{4}+\varepsilon$.

Proof. Let $0<\varepsilon<\frac{1}{4}$ be fixed. We define $f(0)=\frac{3}{4}, f(1)=\frac{1}{4}, f\left(\frac{1}{4}-\varepsilon\right)=\frac{1}{2}=f\left(\frac{3}{4}+\varepsilon\right)$, and $f$ to be linear on the intervening intervals. It follows from theorem 17, [1], that $f \in C^{2}$ and as it is easy to see that $\|f-\phi\|=\left|f\left(\frac{1}{4}-\varepsilon\right)-\phi\left(\frac{1}{4}-\varepsilon\right)\right|=\varepsilon+\frac{1}{4}$ the result follows.

THEOREM 10. If $\|f-\phi\| \leq \frac{1}{4}$ then $f \notin C^{2}$.

Proof. Suppose $\|f-\phi\| \leq \frac{1}{4}, g \in C$, and $f=g^{2}$. Let $g(1)=a$ and $g(0)=b$. We prove a series of five properties of $g$ which lead to the contradiction found in statement six.

Statement 1. $\frac{1}{2} \in g\left(\left[\frac{1}{4}, \frac{3}{4}\right]\right)$.

If $\frac{1}{2} \notin g\left(\left[\frac{1}{4}, \frac{3}{4}\right]\right)$, then as $g$ is continuous, either $g\left(\left[\frac{1}{4}, \frac{3}{4}\right]\right) \subset\left(\frac{1}{2}, 1\right]$ or $g\left(\left[\frac{1}{4}, \frac{3}{4}\right]\right) \subset\left[0, \frac{1}{2}\right)$. For definiteness we suppose the former. Suppose $a \leq \frac{1}{2}$. Then as $g\left(\frac{3}{4}\right)>\frac{1}{2}$ we obtain

$$
\frac{1}{2}<g\left(\frac{1}{2}\right) \in g\left(\left[a, g\left(\frac{3}{4}\right)\right]\right) \subset g\left(g\left(\left[\frac{3}{4}, 1\right]\right)=f\left(\left[\frac{3}{4}, 1\right]\right) .\right.
$$


This contradicts the fact that $f\left(\left[\frac{3}{4}, 1\right]\right) \subset\left[0, \frac{1}{2}\right]$. If $\frac{1}{2} \leq a \leq \frac{3}{4}$, then $g(a) \in\left(\frac{1}{2}, 1\right]$. However, $f(1)=g^{2}(1)=g(a)$ and $f(1) \leq \frac{1}{4}$. Finally, if $a>\frac{3}{4}$, then

$$
\left[\frac{1}{4}, \frac{3}{4}\right] \subset[g(a), a] \subset g\left([a, 1] \subset g\left(\left[\frac{3}{4}, 1\right]\right)\right.
$$

so that

$$
g\left(\left[\frac{1}{4}, \frac{3}{4}\right]\right) \subset g^{2}\left(\left[\frac{3}{4}, 1\right]\right)=f\left(\left[\frac{3}{4}, 1\right]\right) \subset\left[0, \frac{1}{2}\right],
$$

which is untenable. The case when $g\left(\left[\frac{1}{4}, \frac{3}{4}\right]\right) \subset\left[0, \frac{1}{2}\right)$ is similar.

Statement 2. $g(1) \leq \frac{3}{4}$ and $g(0) \geq \frac{1}{4}$.

Suppose $a=g(1)>\frac{3}{4}$. Then

$$
g\left(\left[\frac{1}{4}, \frac{3}{4}\right]\right) \subset g([g(a), a]) \subset g(g([a, 1]))=f([a, 1]) \subset\left[0, \frac{1}{2}\right),
$$

contradicting Statement 1 . The case when $g(0) \geq \frac{1}{4}$ is dispatched similarly.

Statement 3. $g(1)<g(0)$.

Suppose $a=g(1) \geq g(0)=b$. It is easy to see that $a \neq b$ so that we can assume $a>b$. As $a \leq \frac{3}{4}$ it follows that $a \in\left[b, \frac{3}{4}\right]$. As $b \geq \frac{1}{4}$ and $g(b)=f(0) \geq \frac{3}{4}$ we conclude that $\left[b, \frac{3}{4}\right] \subset g([0, b])$. Consequently,

$$
g(a) \in g\left(\left[b, \frac{3}{4}\right]\right) \subset g^{2}([0, b])=f([0, b]) .
$$

Since $g(a) \leq \frac{1}{4}$ and $f(x) \geq \frac{3}{4}-x$ for every $x$ it follows that $b \geq \frac{1}{2}$ and hence $b \in\left[\frac{1}{2}, a\right]$. Therefore,

$$
g(b) \in g\left(\left[\frac{1}{2}, a\right]\right) \subset g^{2}([a, 1])=f([a, 1]) \subset\left[0, \frac{5}{4}-a\right] .
$$

Hence, $\frac{3}{4} \leq \frac{5}{4}-a$ or $a \leq \frac{1}{2}$. From this it follows that $a=b=\frac{1}{2}$ which is a contradiction.

Statement 4. $g(1) \geq \frac{1}{2}$.

Suppose $a=g(1)<\frac{1}{2}$. If $\frac{1}{4} \leq a<\frac{1}{2}$ then $f(1)=g(a) \leq a$, and

$$
\left[\frac{1}{4}, \frac{3}{4}\right] \subset g([a, b]) \subset g([g(a), g(0)]) \subset g^{2}([0, a])=f([0, a]) .
$$

But, $f([0, a]) \subset\left[\frac{3}{4}-a, 1\right]$ and hence $\frac{3}{4}-a \leq \frac{1}{4}$. This implies $a \geq \frac{1}{2}$ which is a contradiction.

Therefore we have $a<\frac{1}{4}$. By Statement $2, b \geq \frac{1}{4}$.

Suppose that $a<\frac{1}{4} \leq b \leq \frac{1}{2}$. If there is an $x \in\left(\frac{1}{2}, 1\right]$ such that $g(x)=b$, then $f(x)=$ $g^{2}(x)=g(b) \geq \frac{3}{4}$ and this is impossible. Also, $g(1)=a<b$ so we obtain that $g\left(\left(\frac{1}{2}, 1\right]\right) \subset$ $[0, b)$. Then

$$
g\left(\left[\frac{1}{4}, b\right]\right) \subset g(g([0, a]))=g^{2}([0, a])=f([0, a]) \subset\left[\frac{3}{4}-a, 1\right] \subset\left(\frac{1}{2}, 1\right] .
$$

In particular, $g\left(\frac{1}{4}\right) \in\left(\frac{1}{2}, 1\right]$. Consequently,

$$
f\left(\frac{1}{4}\right)=g^{2}\left(\frac{1}{4}\right) \in[0, b) \subset\left[0, \frac{1}{2}\right),
$$

which is a contradiction.

Next suppose that $a<\frac{1}{4}$ and $\frac{1}{2}<b<\frac{3}{4}$. Then we have

$$
\begin{aligned}
{\left[\frac{1}{4}, \frac{3}{4}\right] \subset g([a, b]) } & \subset g([g(1), g(b)]) \subset g^{2}([b, 1]) \\
& =f([b, 1]) \subset\left[0, \frac{5}{4}-b\right] .
\end{aligned}
$$

In particular, $\frac{3}{4} \leq \frac{5}{4}-b$ or $b \leq \frac{1}{2}$, which is impossible.

Finally, suppose that $a<\frac{1}{4}$ and $b \geq \frac{3}{4}$. Then

$$
g\left(\left[\frac{1}{4}, \frac{3}{4}\right]\right) \subset g([g(a), b]) \subset g^{2}([0, a])=f([0, a]) .
$$


However,

$$
f([0, a]) \subset\left[\frac{3}{4}-a, 1\right] \subset\left(\frac{1}{2}, 1\right]
$$

and from this it follows that $g\left(\left[\frac{1}{4}, \frac{3}{4}\right]\right) \subset\left(\frac{1}{2}, 1\right]$ thus contradicting Statement 1 .

This final contradiction completes the proof.

Statement 5. $g(0) \leq \frac{1}{2}$.

The proof is analogous to that of the previous Statement. As Statements 3, 4, and 5 are contradictory, this finishes the proof of the theorem.

\section{REFERENCE}

[1] P. D. Humke \& M. Laczkovich. The Borel structure of iterates of continuous functions, Proc. of the Edinburgh Math. Soc. 32 (1989), 483-494. 